\title{
The Network Infrastructure for Radio Astronomy in Italy
}

\author{
Mauro Nanni ${ }^{1}$ \\ INAF - Istituto di Radioastronomia \\ Via Gobetti 101, Bologna, Italy \\ E-mail: m.nannieira.inaf.it \\ Franco Mantovani \\ INAF - Istituto di Radioastronomia \\ Via Gobetti 101, Bologna, Italy \\ E-mail: f.mantovanieira.inaf.it
}

\begin{abstract}
The participation in the EC funded EXPReS project, the agreement with the Gruppo per l'Armonizzazione delle Reti della Ricerca (GARR), the Italian partner of the National Research \& Education Network (NREN), and the agreement with the e-government network of the region Emilia-Romagna made it possible to set up an efficient network infrastructure for Italian radio astronomy.
\end{abstract}

The Medicina 32-m dish is now an active member of the e-EVN array. Here we analyze the progress made since the radio telescope was connected to the regional and GARR backbones in terms of speed in data transmission.

We also present the future plan of the GARR-X network and the opportunities foreseen to connect the Noto 32-m dish and the Sardinia 64-m Radio Telescope to the JIVE hardware correlator and to the software correlator of the Istituto di Radioastronomia.

Science and Technology of Long Baseline Real-Time Interferometry:

The 8th International e-VLBI Workshop - EXPReSO9

Madrid, Spain

June 22-26, 2009

$1 \quad$ Speaker 


\section{Introduction}

We started considering the feasibility of connecting the Italian antennas with optical fibres in 2004. Soon it became clear that the remoteness of radio telescopes from the Point of Presence (PoP) of GARR, the Italian research network, was the problem to overcome.

There are currently three radio telescopes in Italy for Very Long Baseline interferometric observations dedicated to radio astronomy and/or geodynamics. They are located at Medicina, which is $40 \mathrm{~km}$ from the PoP in Bologna, at Noto, $90 \mathrm{~km}$ from the PoP in Catania, and at Matera, $65 \mathrm{~km}$ from the PoP in Bari. Moreover, we also have to consider the Sardinia Radio Telescope, which was under construction $40 \mathrm{~km}$ far from the Cagliari PoP. The so-called "last mile" connection is in our case about 150 miles long in total.

This is not the only difficulty. Analyzing the GARR network specs we are faced with a second severe issue to set up a link for e-VLBI. The PoP in Bologna was one of the four primary network gateways and could easily accommodate another link at a speed of $1 \mathrm{Gbit} / \mathrm{s}$. Also the PoP in Catania could be configured properly. Unfortunately the PoP in Cagliari, which hosts all the scientific and academic traffic of the island of Sardinia, has a speed of $155 \mathrm{Mbit} / \mathrm{s}$ only. The PoP in Bari is in similar conditions.

\section{2. 'Last mile" connection of the antennas}

In the following we briefly describe what has been done in the past few years in order to connect each Italian antenna to the nearest GARR PoP allowing those radio telescopes to take part in e-VLBI observations scheduled by the European VLBI network.

- Medicina: The region of Emilia-Romagna offered us the opportunity to connect the radio telescope with an optical fibre through the network for e-government "Lepida". In order to establish a direct path with the optical fibre between the radio telescope and the PoP in Bologna, it was necessary to cross a regional railway. Unfortunately this caused bureaucratic problems that delayed the link.

At the end of 2005 a connection was established through a backup loop path of $120 \mathrm{~km}$ length, which needed an intermediate repeater (a cheap switch). That link allowed us to become an active partner of the EXPReS project and participate in e-VLBI sessions of the EVN. We spent the next two years (in cooperation with the GARR and JIVE staff) to increase the speed from the original $300 \mathrm{Mbit} / \mathrm{s}$ to the current $998 \mathrm{Mbit} / \mathrm{s}$. In the period we took part in all the eVLBI sessions that were scheduled. Finally, on April 2009, the railway problem was solved and a connection with a "dark fibre" established. We are in the process of acquiring 10-Gbit/s optical transceivers for the connection from Medicina to Bologna. However, the 10-Gbit/s light path from Bologna to the JIVE correlator will only be available when the next GARR backbone is activated. This is expected to happen in 2010 .

- Noto: The Noto telescope is by far the remotest of the antennas of the Istituto di Radioastronomia. However, Noto Observatory is not the only institution requiring an optical 
fibre in the south-eastern part of Sicily. The National Institute of Nuclear Physics (INFN) is also interested to have a link between Catania and the experimental station of the NEutrino Mediterranean Observatory (NEMO) project. Both institutions spent quite some time finding out if there were fibres available in that part of Sicily, without success. Only recently we were able to obtain map of the available infrastructure and information about the plan for a regional Network. GARR has included both connections to the Noto radio telescope and to the NEMO station in a call for tender. Two network operators made offers. The expected cost is in the range of 250 to $500 \mathrm{k} €$, but it is not clear when these funds will be available.

- SRT: The Sardinia Radio Telescope (SRT) is under construction and should be completed by the end of 2010. With a Regional Order on the 18-3-2008, the region of Sardinia allocated $2 \mathrm{M} €$ for the implementation of the research network, which includes the connection of SRT with the PoP in Cagliari. From April this year, a couple of 2.5-Gbit/s lambda switches of a public operator are available for the GARR connection of the island of Sardinia to the continent. Moreover, in the next years the Janna consortium (a consortium with the participation of the Region of Sardinia) will connect Sardinia with both the continent and Sicily with two new undersea cables. It is planned that fibres will be made available on those cables for the scientific network.

- Matera: The Matera dish, run by the National Space Agency (ASI), is at present connected to the Bari PoP by a 50-Mbit/s link. ASI is currently considering the activities of the Centro di Geodesia Spaziale Giuseppe Colombo and the role of the 20-m VLBI dish.

\section{GARR-X: The new network for scientific applications}

GARR-X is the plan for the next six years of the Italian research network. The project foresees long term leasing of optical fibres (both the backbone and accesses fibres) that will be under the complete control of GARR. GARR will acquire and install next generation equipments, like routers and photonics, to allow a high level of reconfiguration with multilambda capacity on the same fibre pair at a speed of 10,40 and $100 \mathrm{Gbit} / \mathrm{s}$.

The Phase 0 of GARR-X started with a call for tender in February 2009. The plan is to connect at least $40 \%$ of the existing sites before Summer 2010 (see Fig. 1). The budget for Phase 0 is of about $27 \mathrm{M€}$.

A new network infrastructure will be built in the next few months in Bologna, the "Lepida Metropolitan Area Network (MAN)". This is a metropolitan area network realized with seven independent layers on the same cables that will connect municipality, provincial and regional offices, province offices, hospitals, university departments, police commissariats, and research institutes via private optical networks for a total of more of 350 nodes. 


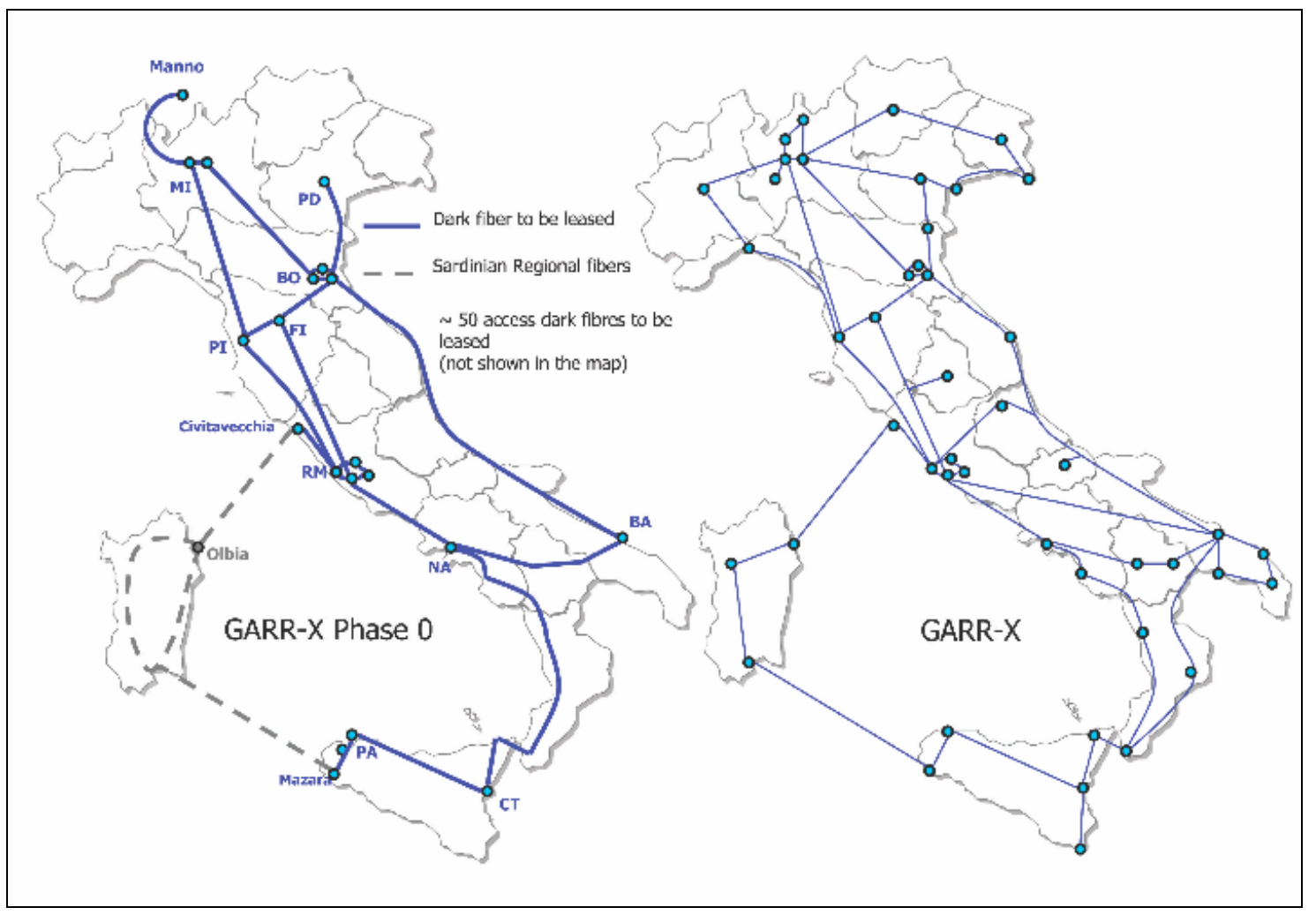

Fig. 1 - The links provided in Phase 0 (left) and the complete GARR-X infrastructure (right). Courtesy of Massimo Carboni.

Thanks to this local infrastructure GARR plans to set up 3 primary PoP nodes in Bologna. One of them will be put in the scientific campus where the headquarters of the INAF-Istituto di Radioastronomia are located. The institute will become the PoP of the Italian radio astronomy network.

\section{An Optical Private Network for radio astronomy}

The new network infrastructure that will be made available in the next years will allow us to set up an Optical Private Network for radio astronomy in Italy (Fig. 2). Such a network will allow us not only to take part in global e-VLBI observations, or to be involved in international projects like LOFAR, but also to set up a VLBI network which will form a new special-purpose observing facility open to the astronomical community.

We have estimated the actual annual costs for optical links in a basic configuration of the network for radio astronomy that can be set up in the next two years (Table 1). The total cost thus amounts to about $90 \mathrm{k} € /$ year. An annual cost of about $200 \mathrm{k} € /$ year can be estimated for optical links at $10 \mathrm{Gbit} / \mathrm{s}$ for SRT and Noto, and to close the loop to the islands with Janna cables. 


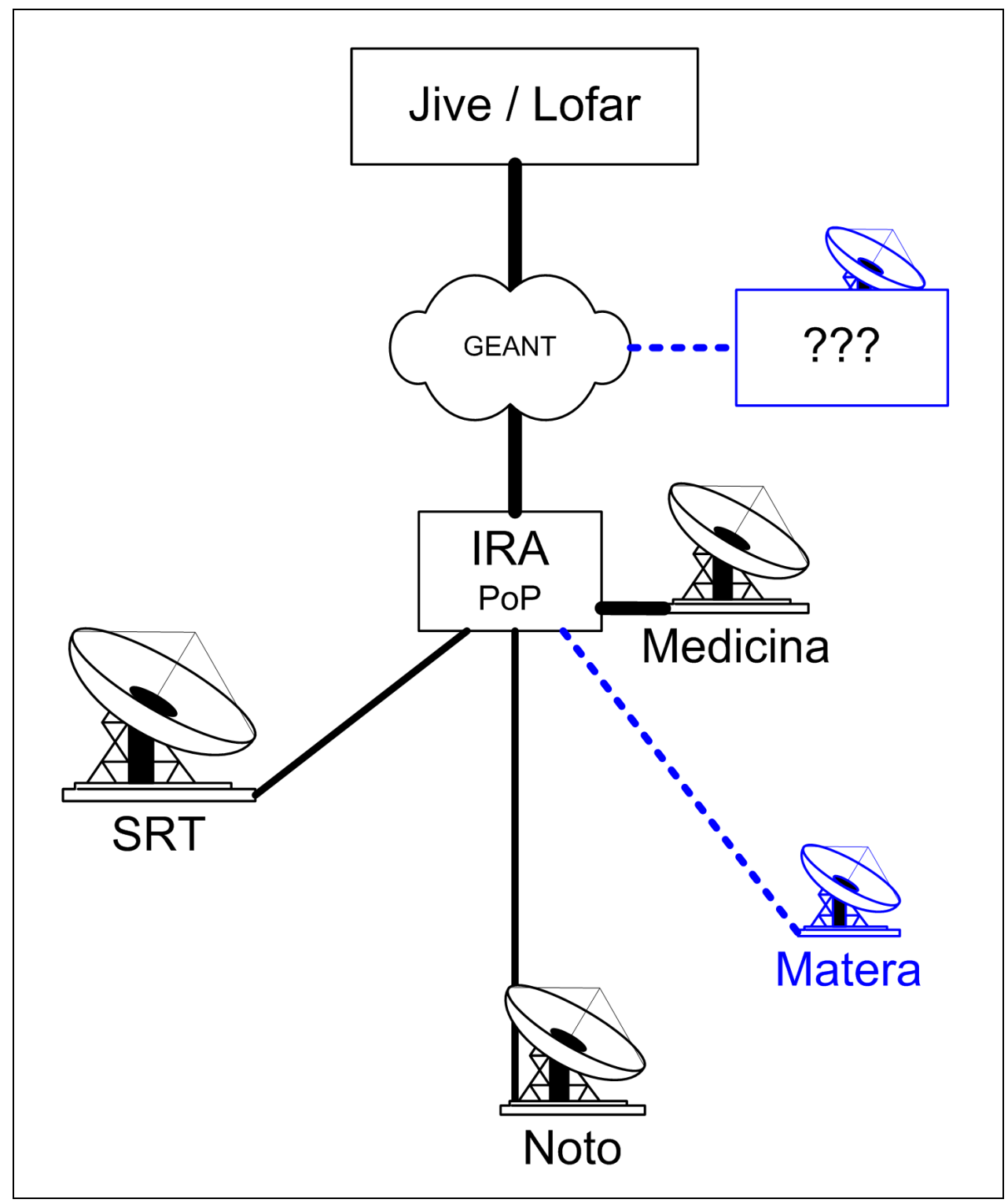

Fig. 2 - The Optical Private Network for radio astronomy in Italy.

\begin{tabular}{|l|c|c|}
\hline \multicolumn{3}{|c|}{ Annual Costs } \\
\hline Link & Speed & $\mathrm{k} €$ \\
\hline IRA - JIVE/Lofar & $10 \mathrm{Gbit}$ & 50 \\
\hline IRA - Medicina & $10 \mathrm{Gbit}$ & 0 \\
\hline IRA - Noto & $1 \mathrm{Gbit}$ & 20 \\
\hline IRA - SRT & $1 \mathrm{Gbit}$ & 20 \\
\hline
\end{tabular}

Table 1: Estimated costs for basic configuration 
Obviously, these costs are calculated on current prices that hopefully will become cheaper in the coming years. Moreover, the GARR network will allow the usage of lambda switches to set up connections on demand to other antennas and correlators of the international optical fibre network.

\section{Software Correlator}

To complete the project of an Italian VLBI network we are deeply involved in development studies of the DiFX software correlator [1] together with other radio astronomy groups. Several installation and correlation tests were made by John Morgan in the course of his a Ph.D. project at the Institute of Radioastronomy. A complete pipeline to process EVN data on a cluster of eight $1 \mathrm{U}$ computers was set up. Further tests are underway on a new Dell blade M1000 system, which has 96 cores and a 10-Gbit/s internal switch that connects the blades.

The goal is a real time correlator that can process 4 to 6 stations at a rate up to $1 \mathrm{Gbit} / \mathrm{s}$. We are not close to that target, but we are gaining experience with the correlation process. Observations with data recorded by the Medicina, Matera, Effelsberg, and Wettzell antennas were correlated with success. Moreover, large computational resources will be made available at the SRT station. The future high-speed connection will allow us to realize distributed cluster correlation.

\section{Conclusions}

The next two years will be critical for the development of the Italian e-VLBI network. Medicina will be connected at $10 \mathrm{Gbit} / \mathrm{s}$. With the development of the MAN in Bologna and the GARR-X optical network all the Italian antennas will be connected and ready for e-VLBI observations.

In the coming months the main problem to be solved is to achieve the "last mile" to Noto.

With the implementation of a software correlator we will provide a national network for eVLBI that can be used for observing projects like source monitoring, spectral line astrometry, radio transients, and geodesy.

The FP6 EXPReS project was a great opportunity to work with people of the radio astronomical community to set up the e-VLBI infrastructure. We hope that this activity will be continued with FP7 NEXPReS.

\section{References}

[1] A.T. Deller, S.J.Tingay, M.Bailes and C.West DiFX: A Software Correlator for Very Long Baseline Interferometry Using Multiprocessor Environments, 2007 PASP, 119, 318 